Radiologe 2020 $60: 42-47$

https://doi.org/10.1007/s00117-019-00611-2

Online publiziert:21. November 2019

(C) Der/die Autor(en) 2019

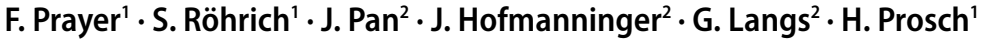

'Universitätsklinik für Radiologie und Nuklearmedizin, Medizinische Universität Wien, Wien, Österreich ${ }^{2}$ Computational Imaging and Research Lab, Universitätsklinik für Radiologie und Nuklearmedizin, Medizinische Universität Wien, Wien, Österreich

\title{
Künstliche Intelligenz in der Bildgebung der Lunge
}

\begin{abstract}
Künstliche Intelligenz (KI) stellt seit einigen Jahren einen der Forschungsschwerpunkte in der Thoraxradiologie dar. Mithilfe von Algorithmen sollen in Zukunft Detektion, Quantifizierung, Charakterisierung und Verlaufsprädiktion von Lungenpathologien in der Computertomographie (CT) verbessert werden. In diesem Übersichtsartikel werden die neuesten Entwicklungen im Bereich der künstlichen Intelligenz in der CT der Lunge mit Fokus auf pulmonale Rundherde und interstitielle Lungenerkrankungen beleuchtet.
\end{abstract}

\section{Interstitielle \\ Lungenerkrankungen}

Interstitielle Lungenerkrankungen beinhalten ein weites Spektrum möglicher Erkrankungen unterschiedlicher Prognose. Um eine optimale Behandlung zu ermöglichen, werden klinische, laborchemische, histologische und radiologische Informationen im Rahmen eines multidisziplinären Boards diskutiert [1]. Da eine Lungenbiopsie aufgrund des erhöhten Risikos in dieser Patientenpopulation oft nicht möglich und die Klinik besonders zu Beginn der Erkrankung unspezifisch ist, hat die Radiologie in Form der hochauflösenden CT in den letzten Jahrzehnten deutlich an Bedeutung gewonnen [2].

Primäre Aufgabe des Radiologen ist es, in der CT das Muster einer "gewöhnlichen interstitiellen Pneumonie“(„usual interstitial pneumonia“, UIP) zu erkennen oder auszuschließen (• Abb. 1). Bei passender Klinik erlaubt die Detektion der UIP die Diagnose der idiopathischen Lungenfibrose (,idiopathic pulmonary fibrosis", IPF) ohne die Notwendigkeit einer Lungenbiopsie [3, 4]. Allerdings besteht selbst unter erfahrenen Thoraxradiologen nur eine mittelmäßige Übereinstimmung für die Erkennung des Honigwabenmusters ("honeycombing"), welches zentraler Bestandteil der UIP ist [5]. Zur objektiven und zeitnahen Diagnostik interstitieller Lungenerkrankungen ist deshalb eine Unterstützung durch KI wünschenswert.

Bei der vorrangig verwendeten Form der KI, dem maschinellen Lernen, werden zwei grundlegende Ansätze unterschieden. Beim überwachten maschinellen Lernen stützt sich die Entwicklung eines Algorithmus auf durch Experten aufbereitete Daten, z. B. durch Radiologen im CT-Bild markierte Areale wie Tumor- oder Fibrosegewebe ([6]; - Abb. 2). Dieser Prozess erlaubt einerseits das Erlernen spezifischer Muster auf Basis einer moderaten Menge von Trainingsdaten, ist jedoch aufgrund der zeitintensiven Markierung durch Radiologen mit spezifischer Expertise sehr ressourcenaufwändig und beinhaltet potenziell Fehler infolge falscher Markierungen etc. Auf der anderen Seite erfordert unüberwachtes Lernen keine vorausgehende Markierung pathologischer Areale. Der Algorithmus wird trainiert, selbstständig Merkmale in Daten zu identifizieren, aufgrund derer verschiedene Gruppen unterschieden werden können (• Abb. 3). Dem Potenzial, bisher unbekannte - d.h., visuell schwer zu erfassende - Biomarker zu finden, steht die große, für unüberwachtes Lernen erforderliche Datenmenge gegenüber [7]. Maschinelles Lernen wird be- reits mit vielversprechendem Erfolg in der CT von interstitiellen Lungenerkrankungen eingesetzt [8].

\section{Maschinelles Lernen zur Unterstützung der Diagnose von Lungenfibrose}

Deep Learning bezeichnet eine Form von maschinellem Lernen, bei welchem häufig neuronale Netzwerke eingesetzt werden [9]. Mithilfe eines solchen neuronalen Netzwerks zeigte Anthimopoulos' Gruppe bereits 2016, dass es möglich ist, gesundes Lungengewebe sowie sechs verschiedene interstitielle Erkrankungsmuster (Milchglas, Retikulation, Mischung von Milchglas und Retikulation, kleine Rundherde, Konsolidierung und Honigwabenmuster) mit einer Genauigkeit von $85,5 \% \mathrm{zu}$ unterscheiden [10]. Im Jahr 2018 konnten Kims Gruppe die Genauigkeit ihres Algorithmus zur Unterscheidung sechs verschiedener interstitieller Erkrankungsmuster v.a. durch verbesserte Differenzierung ähnlicher Muster, wie Honigwabenmuster und Retikulation, auf bis zu 95,1\% steigern [11]. Für diese Ansätze sind jedoch durch Radiologen segmentierte Regionen mit spezifischen CTMustern notwendig und beinhalten dadurch potenziell untersucherabhängige Fehler. Mit vergleichsweise geringerer Genauigkeit (68,6\%), jedoch ohne die Notwendigkeit einer vorhergehenden, aufwändigen Segmentierung, gelang es Gao et al., Muster mithilfe eines neuronalen Netzwerks in vollständigen CTSchnitten zu identifizieren [12]. Walsh et al. entwickelten als Erste einen Algorithmus, welcher, den ATS/ERS/JRS/ 


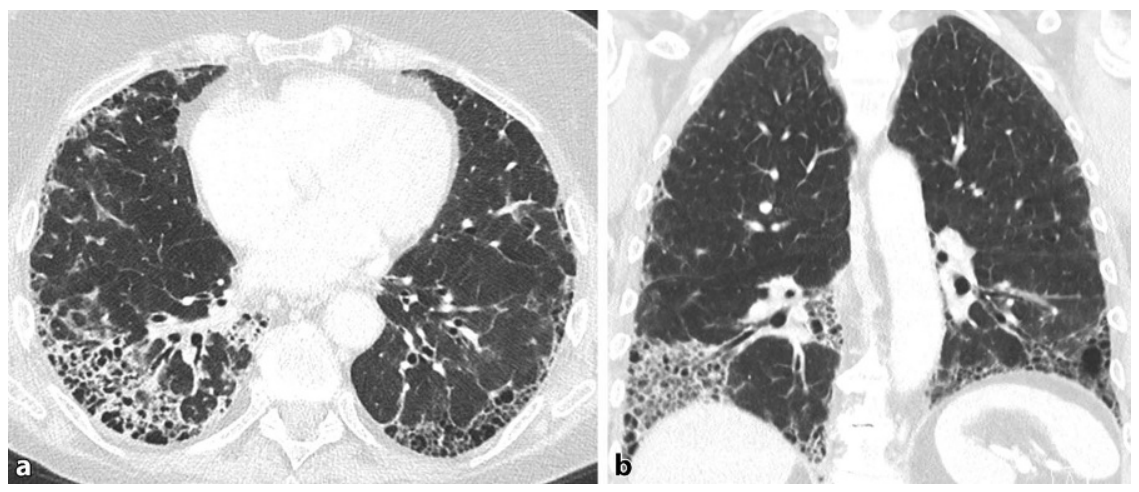

Abb. $1 \Delta$ Axiale (a) und koronare (b) Rekonstruktionen einer hochauflösenden CT (HRCT) des Thorax bei einer 74-jährigen Patientin mit Ruhedyspnoe. CT-Muster einer gewöhnlichen interstitiellen Pneumonie ("usual interstitial pneumonia“, UIP) mit basal betontem Honigwabenmuster (,honeycombing"), Traktionsbronchiektasien sowie subpleuralen retiklären Verdichtungen. Die Erkennung des Honigwabenmusters unterliegt einer deutlichen Interobserver-Varaibilität

ALAT-Leitlinien für die Diagnostik der IPF [13] entsprechend, die CT Muster „UIP“, „mögliche UIP“ und „,inkonsistent mit UIP“ in wenigen Sekunden mit höherer Genauigkeit $(73,3 \%)$ unterscheidet als erfahrene Thoraxradiologen $(70,7 \%$; [8]). Christie et al. konnten zeigen, dass ein computergestütztes System (INTACT-System) IPF mit einer ähnlichen Genauigkeit wie Radiologen in CT-Bildern erkennen kann [14]. Diese Studien zeigen das Potenzial von DeepLearning-Algorithmen, Expertise in Bezug auf spezifische Fragestellungen (in diesem Fall UIP vs. keine UIP in der CT) mithilfe überschaubarer Ressourcen in Zukunft in Sekundenschnelle an Orten zu nutzen, wo diese bisher nicht verfügbar war.

\section{Quantitative CT zur Bestimmung der Ausdehnung von Lungenfibrosen}

Quantitative CT bezeichnet verschieden Methoden der Analyse von CT-Daten, welche auf Parametern beruhen, die der visuellen Beurteilung nicht zugänglich sind, wie z.B. Histogramm- und Texturanalysen [15]. Mithilfe der quantitativen Computertomographie kann das Ausmaß von Lungenveränderungen objektiviert und somit die Dynamik der Pathologien zwischen Untersuchungen zu verschiedenen Zeitpunkten beurteilt werden [16]. Ein Zugang zur Detektion und Quantifizierung von Lungenpathologien stellt die Analyse basierend auf
Dichtewerten des Lungengewebes dar. Ein Lungenemphysem kann anhand niedriger Dichtewerte (z.B. $-800 \mathrm{HU}$ ) automatisiert erfasst und gleichzeitig quantifiziert werden. Müller et al. konnten mithilfe dieser Methode schon 1988 eine Korrelation des EmphysemausmaBes, basierend einerseits auf quantitativer CT sowie auf Lungenpräparaten nach Lobektomie, zeigen [17]. Nach diesem Prinzip ist es ebenfalls möglich, anhand von erhöhten Lungendichtewerten interstitielle Lungenerkrankungen $\mathrm{zu}$ detektieren und die Dynamik zwischen Untersuchungen zu beurteilen. Ohkubo et al. konnten zeigen, dass in IPF-Patienten der Prozentanteil von normalem Lungengewebe, definiert als Gewebe mit -950 HU bis -701 HU, bzw. von Gewebe mit pathologisch erhöhter Dichte gegenüber dem Gesamtlungengewebe mit der forcierten Vitalkapazität korreliert und mittelschwere sowie schwere Fälle von IPF identifizieren kann [18]. Während die Densitometrie eine verlässliche Methode zur Analyse globaler Lungenveränderungen darstellt, ist sie jedoch weniger geeignet in Bezug auf subtile oder spezifische lokale Erkrankungsmuster, welche die Prognose beeinflussen können, wie Traktionsbronchiektasien oder lokalisiertes Honigwabenmuster. Aus diesen Gründen bringt die Densitometrie alleine keine verbesserte Prädiktion des Überlebens bei interstitiellen Lungenerkrankungen [19]. Maschinelles Lernen ermöglicht zunehmend die Identifikation von Texturmerkmalen für quantita- tive CT-Analysen, ein Zugang, welcher bereits 1997 für die Charakterisierung interstitieller Lungenerkrankungen exploriert wurde [20]. Die Kombination von Textur- und Dichtemerkmalen spiegelt die visuelle Beurteilung durch den Radiologen und führt zu einer verbesserten Prädiktion des Outcomes. In einer rezenten Studie von Kim et al. an Patienten mit idiopathischer Lungenfibrose war eine Verschlechterung des texturbasierten quantitativen LungenfibroseScores nach 6 Monaten um zumindest $4 \%$ mit einer visuell erfassbaren Verschlechterung nach 12 Monaten sowie mit einer Reduktion des progressionsfreien Überlebens assoziiert [21].

Eine auch kommerziell vertriebene Anwendung der quantitativen CT ist CALIPER (Computer Aided Lung Informatics for Pathology Evaluation and Rating), ein Algorithmus, der die Klassifikation von Lungengewebe in verschiedene Klassen basierend auf Dichteund Texturmerkmalen auf Voxelebene erlaubt. Bei Patienten mit IPF kann die computergestützte CT-Auswertung die Schwere der Lungenveränderungen genauer schätzen als visuelle Scores, wobei das durch CALIPER gemessene Ausmaß besser mit Lungenfunktionsparametern korreliert [22]. Jacob et al. konnten des Weiteren zeigen, dass eine CALIPERbasierte CT-Auswertung eine genauere Prädiktion der Mortalität erlaubt als visuelle Scores [23]. Darüber hinaus konnte mit dem pulmonalen Gefäßvolumen ein neuer Parameter beschrieben werden, welcher einerseits visuell kaum quantifizierbar ist und die Volumenerhöhung andererseits mit Mortalität assoziiert ist. Dies ist ein Beispiel für das Potenzial quantitativer CT-Analysen, Merkmale in CT-Bildern zu identifizieren, die entweder der Beurteilung durch den Radiologen entgehen (bzw. für eine visuelle Quantifizierung zu sperrig sind) oder bisher nicht bekannt waren, aber die dennoch den diagnostischen bzw. prognostischen Wert des radiologischen Befunds steigern können. 


\section{Radiomics und maschinelles Lernen in der Abklärung von Rundherden}

\section{Detektion pulmonaler Rundherde}

Vor allem seit der Einführung des Lungenkarzinom-Screenings in den USA und anderen Ländern steigt auch der Bedarf an Software zur Verbesserung der Detektion von Rundherden („computer-aided detection“, CAD), und entsprechende Lösungen sind bereits seit einigen Jahren im klinischen Einsatz. Die Sensitivität konventionell programmierter, nicht auf maschinellem Lernen basierter CAD-Software reicht von 54 bis $95 \%$ [24]. Durch den Einsatz von modernen, auf maschinellem Lernen basierten Ansätzen konnte die Sensitivität von CAD-Software auf 83,1-96,6\% gesteigert werden [25]. Die Detektion von Lungenrundherden kann weiter verbessert werden, wenn zum Lernen neuronale Netzwerke (Convolutional Neural Network, ConvNet) herangezogen werden. Mit Hilfe von ConvNets lässt sich eine Genauigkeit von $82,2-97,6 \%$ bei einer Sensitivität von $83,1-96,6 \%$ und einer Spezifität von $71,4-98,2 \%$ erreichen [26].

\section{Klassifikation pulmonaler Rundherde}

Die Risikoabschätzung von Rundherden und Raumforderungen war einer der ersten Ansätze bei denen versucht wurde, mittels Computerunterstützung die Abschätzung der Prognose zu verbessern. Bereits 1963 veröffentlichte Gwilym Lodwick eine Arbeit, in der er an Röntgenbildern des Thorax semantische Merkmale wie Größe, Lage, Begrenzung und Dichte von 543 Lungenkarzinomen durch numerische Werte beschrieb und mit Hilfe eines Computers die Prognose der Patienten abschätzte [27].

Mit Hilfe von CT-Bildern lassen sich Rundherde, außer durch semantische Merkmale, computerunterstützt auch durch komplexere Form- und Texturmerkmale erfassen. Rundherde können auf diese Weise durch mehr als hundert verschiedene Texturmerkmale beschrieben werden. Die automatische Erfassung

Radiologe 2020 -60:42-47 https://doi.org/10.1007/s00117-019-00611-2

(c) Der/die Autor(en) 2019

F. Prayer · S. Röhrich · J. Pan · J. Hofmanninger · G. Langs · H. Prosch

Künstliche Intelligenz in der Bildgebung der Lunge

\section{Zusammenfassung}

Klinisches/methodisches Problem. Künstliche Intelligenz (KI) hat das Potenzial, durch automatisierte Detektion, Quantifizierung, Klassifikation und Verlaufsprädiktion die Diagnostik und Behandlung von Patienten mit Lungenerkrankungen zu verbessern. Radiologische Standardverfahren. Interstitielle Lungenerkrankungen stellen aufgrund unspezifischer Symptomatik, geringer Anzahl visuell erfassbarer computertomographischer Erkrankungsmuster sowie der potenziell schwerwiegenden Prognose einen Forschungsschwerpunkt für KI dar. Methodische Innovationen. Durch überwachtes und unüberwachtes maschinelles Lernen können Erkrankungsmuster in der $\mathrm{CT}$ anhand von Merkmalen identifiziert und Zusammenhänge mit bestimmten Erkrankungen und deren Verlauf analysiert werden.

Leistungsfähigkeit. Maschinelles Lernen verbessert einerseits die automatisierte Detektion pulmonaler Rundherde. Andererseits bietet es die Möglichkeit, diese Rundherde zu charakterisieren, was besonders im Hinblick auf Lungenkrebs-Screening-Programme Ressourceneffizienz verspricht.

Bewertung. Neben der Notwendigkeit leistungsstarker Algorithmen stellen die Daten, anhand welcher die KI Erkrankungsmuster lernt, eine Herausforderung dar. Notwendig sind aufwändige Expertenannotation sowie eine ausreichende Größe der Trainingsdatensätze, um physiologische und pathologische Diversität abzubilden. Aufgrund der noch fehlenden Standardisierung in diesem jungen Forschungsfeld stellen sich Fragen der Vergleich- und Reproduzierbarkeit. Empfehlung für die Praxis. Dieser Übersichtartikel beschäftigt sich mit dem State of the Art und den Herausforderungen von $\mathrm{KI}$ in der Bildgebund der Lunge unter besonderer Berücksichtigung von interstitiellen Lungenerkrankungen und der Detektion und Beurteilung von Lungenrundherden.

Schlüsselwörter

Thorax · Deep Learning · Computertomographie · Lungenkrebs · Interstitielle Lungenerkrankung

\section{Artificial intelligence in lung imaging}

\section{Abstract}

Clinical/methodical issue. Artificial intelligence (Al) has the potential to improve diagnostic accuracy and management in patients with lung disease through automated detection, quantification, classification, and prediction of disease progression.

Standard radiological methods. Owing to unspecific symptoms, few well-defined CT disease patterns, and varying prognosis, interstitial lungs disease represents a focus of Al-based research.

Methodical innovations. Supervised and unsupervised machine learning can identify CT disease patterns using features which may allow the analysis of associations with specific diseases and outcomes.

Performance. Machine learning on the one hand improves computer-aided detection of pulmonary nodules. On the other hand it enables further characterization of pulmonary nodules, which may improve resource effectiveness regarding lung cancer screening programs.
Achievements. There are several challenges regarding $\mathrm{Al}$-based $\mathrm{CT}$ data analysis. Besides the need for powerful algorithms, expert annotations and extensive training data sets that reflect physiologic and pathologic variability are required for effective machine learning. Comparability and reproducibility of Al research deserve consideration due to a lack of standardization in this emerging field.

Practical recommendations. This review article presents the state of the art and the challenges concerning $\mathrm{Al}$ in lung imaging with special consideration of interstitial lung disease, and detection and consideration of pulmonary nodules.

Keywords

Thorax · Deep learning · Computed tomography · Lung cancer · Interstitial lung disease 


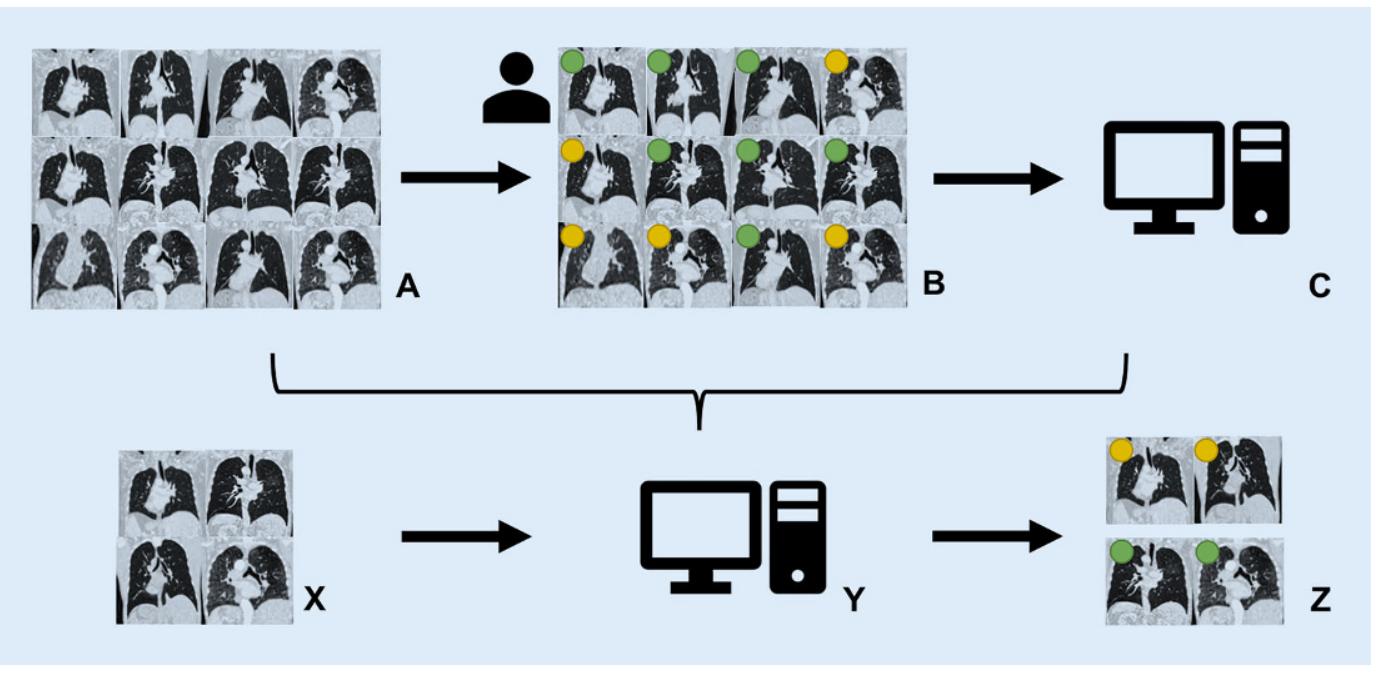

Abb. 2 \ Überwachtes maschinelles Lernen benötigt Datensätze $(A)$, welche eine bestimmte Pathologie beinhalten. Experten markieren in diesem Datensätzen Areale von Interesse bzw. teilen Fälle in Gruppen ein (krank und gesund, benigne und maligne, Krankheit A und Krankheit B, etc.) (B). Anhand dieser Annotationen wird ein Algorithmus trainiert (C), Merkmale in den Bilddaten zu identifizieren, durch welche Areale von Interesse bzw. die verschiedenen Gruppen unterschieden werden können. Mithilfe des entwickelten Algorithmus $(Y$ ) können nun die gelernten Merkmale dazu eingesetzt werden, in nicht annotierten Bilddaten $(X)$ Areale von Interesse oder verschiedene Gruppen zu identifizieren $(Z)$

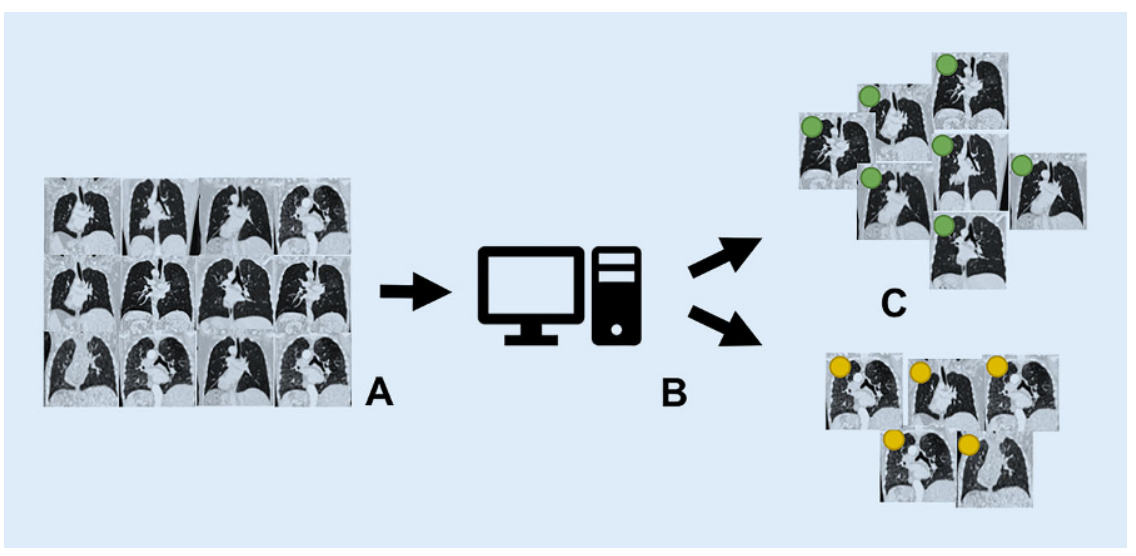

Abb. 3 ॥ Unüberwachtes maschinelles Lernen benötigt sehr umfangreiche Datensätze $(A)$, um die gesamte Variation einer Pathologie bzw. der Physiologie abzubilden. Ein Algorithmus $(B)$ kann nun ohne vorausgehende Annotation trainiert werden, Gruppen in diesen Daten zu identifizieren (C). Auf diese Weise können potenziell neue Bildgebungsmerkmale für die Detektion und Charakterisierung von Lungenpathologien in der CT identifiziert werden

und Analyse einer großen Zahl von quantitativen Bilddaten wird heute als Radiomics bezeichnet.

Ein typischer Radiomics-Arbeitsablauf wird dabei grob in dreiSchritte unterteilt: die Bildakquisition und Segmentation, die Erfassung von Texturmerkmalen und die Prognoseabschätzung [28].

\section{Bildakquisition und \\ Segmentierung}

Voraussetzung für Radiomics-Studien sind Bilddaten, die reproduzierbar analysiert werden können. Dementsprechend nehmen die Bildakquisition sowie die Rekonstruktion der Bilder eine zentrale Rolle ein, da Radiomics-Studien anfällig für variable Untersuchungsprotokolle sind.

Die zu analysierenden Rundherde müssen in einem ersten Schritt segmentiert werden. Da eine manuelle Segmentation nur beschränkt reproduzierbar ist, sollten Rundherde (semi)-automatisch segmentiert werden. Neue Ansätze der automatischen Segmentierung, die auf ConvNets beruhen, sind hier deutlich genauer als bisher verwendete Algorithmen [29].

\section{Erfassen von Texturmerkmalen}

Nach der Segmentierung werden mehrere hundert Texturmerkmale der Rundherde automatisch extrahiert. Diese Radiomics-Merkmale können grob in strukturelle Merkmale (z.B. Form und Volumen), Intensitätsmerkmale (graphische Repräsentation der Intensitätsverteilung), Texturmerkmale (räumliche Beziehung zwischen Voxeln) und Wavelet-Merkmale (komplexere Analyse der Frequenzbereiche) unterteilt werden [28]. Diese Gruppen von Merkmalen sind dabei unterschiedlich anfällig für verschiedene Untersuchungsprotokolle und der Bildrekonstruktion (z.B. Schichtdicke, Kernel).

Die große Zahl an verschiedenen Radiomics-Merkmalen muss in einem weiteren Schritt auf eine überschaubare Zahl reduziert werden. Zu diesem Zweck werden redundante, abhängige und instabile Merkmale ausgeschlossen.

\section{Vorhersagemodelle - Klassifikati- on von Rundherden}

Die Radiomics-Merkmale werden in einem letzten Schritt für die Entwicklung von Vorhersagemodellen herangezogen. Jedoch ist die Zahl der für die Entwick- 
lung dieser Modelle herangezogenen Datenpunkte in der Regel relativ klein. Dieser Umstand, zusammen mit der ausgeprägten Heterogenität der Daten, führt zu einer schlechten Übertragbarkeit dieser Modelle auf Routinedaten [29].

Die Klassifikation von Lungenrundherden kann jedoch durch ConvNets deutlich verbessert werden. Erste Studien konnten zeigen, dass sich durch ConvNets vor allem die Zahl der falschpositiven Resultate reduzieren lässt, was gerade im Lungenkrebs-Screening von wesentlicher Bedeutung ist [29].

\section{Radiogenomics}

Radiomics-Merkmale können neben der Klassifikation von Rundherden auch zur Vorhersage von bestimmten genetischen Mutationen in Lungenkarzinomen verwendet werden, so z. B. Mutationen des Epidermal-Growth-FactorRezeptors (EGFR) oder des Kirsten RAt Sarcoma virus Oncogens (K-RAS) bei nichtkleinzelligen Lungenkarzinomen $[30,31]$.

\section{Herausforderungen und Ausblick}

Ein effektiver Einsatz KI-basierter Bildanalysen setzt sowohl Expertise bezüglich der verwendeten Algorithmen als auch eine hohe Datenqualität voraus. Der Aufbau von Datensätzen, welche die große Bandbreite von Pathologien, aber auch von physiologischer Variation beinhalten und (z.B. histologisch) gesicherte Information bieten, sind mit einem erheblichen Ressourcenaufwand verbunden und in manchen Fällen nicht an einem einzelnen Zentrum realisierbar. Hinzu kommt die Variabilität aufgrund technischer Gegebenheiten, wie verschiedener CT-Scanner oder Datenrekonstruktionen, die für die Auswertung berücksichtigt werden muss.

Während manche dieser Probleme durch die Weiterentwicklung im Feld der Computerwissenschaften behoben werden können, kommt der Anpassung von Arbeitsabläufen und Protokollen eine erhebliche Bedeutung zu. Ein wichtiges Vorhaben wäre die Standardisierung von radiologischen Bilddaten auf nationaler und internationaler Ebene, um com- puterbasierten Zugriff und Auswertung zu vereinfachen bzw. überhaupt erst zu ermöglichen.

Den Herausforderungen gegenüber steht das Potenzial, durch eine qualitativ hochwertige automatisierte Bildauswertung Expertenwissen und visuell nichterfassbare, prädiktive Biomarker weithin verfügbar zu machen und den Radiologen darin zu unterstützen, den diagnostischen Wert der CT zu maximieren.

\section{Fazit für die Praxis}

- Maschinelles Lernen verspricht eine verbesserte Abschätzung der Prognose von fibrosierenden Lungenerkrankungen und eine verbesserte Charakterisierung von pulmonalen Rundherden.

- Für die klinische und weitverbreitete Integration der computerbasierten Analyse ist eine verbesserte Standardisierung der radiologischen Bilddaten nötig.

Assoc. Prof. PD Dr. H. Prosch
Universitätsklinik
für Radiologie und
Nuklearmedizin, Medizinische
Universität Wien
Währinger Gürtel 18-20,
1090 Wien, Österreich
helmut.prosch@
meduniwien.ac.at

Funding. Open access funding provided by Medical University of Vienna.

\section{Einhaltung ethischer Richtlinien}

Interessenkonflikt. F. Prayer, S. Röhrich, J. Pan, J. Hofmanninger, G. Langs und H. Prosch: Institutionelle Kooperation mit Siemens Healthineers. H. Prosch: Unrestricted Research Grant von Boehringer Ingelheim; Forschungsunterstützung von Nvidia.

Für diesen Beitrag wurden von den Autoren keine Studien an Menschen oder Tieren durchgeführt. Für die aufgeführten Studien gelten die jeweils dort angegebenen ethischen Richtlinien.

Open Access. Dieser Artikel wird unter der Creative Commons Namensnennung 4.0 International Lizenz (http://creativecommons.org/licenses/by/4.0/deed. de) veröffentlicht, welche die Nutzung, Vervielfäl- tigung, Bearbeitung, Verbreitung und Wiedergabe in jeglichem Medium und Format erlaubt, sofern Sie den/die ursprünglichen Autor(en) und die Quelle ordnungsgemäß nennen, einen Linkzur Creative Commons Lizenz beifügen und angeben, ob Änderungen vorgenommen wurden.

\section{Literatur}

1. Raghu G, Remy-Jardin M, Myers JL, Richeldi L, Ryerson CJ, Lederer DJ, Behr J, Cottin V, Danoff SK, Morell F, Flaherty KR, Wells A, Martinez FJ, Azuma A, Bice TJ, Bouros D, Brown KK, Collard HR, Duggal A, Galvin L, Inoue Y, Jenkins RG, Johkoh T, Kazerooni EA, Kitaichi M, Knight SL, Mansour G, Nicholson AG, Pipavath SNJ, Buendia-Roldan I, Selman M, Travis WD, Walsh S, Wilson KC, E. R. S. J. R. S. American Thoracic Society, S. Latin American Thoracic (2018) Diagnosis of idiopathic pulmonary fibrosis. An official ATS/ERS/JRS/ALAT clinical practice guideline. Am J Respir Crit Care Med 198:e44-e68. https://doi.org/10.1164/rccm. 201807-1255ST

2. Jacob J, Hansell DM (2015) HRCT of fibrosing lung disease. Respirology 20:859-872. https://doi.org/ 10.1111/resp. 12531

3. Travis WD, Costabel U, Hansell DM, King TE Jr., Lynch DA, Nicholson AG, Ryerson CJ, Ryu JH, Selman M, Wells AU, Behr J, Bouros D, Brown KK, Colby TV, Collard HR, Cordeiro CR, Cottin V, Crestani B, Drent M, Dudden RF, Egan J, Flaherty K, Hogaboam C, Inoue Y, Johkoh T, Kim DS, Kitaichi M, Loyd J, Martinez FJ, Myers J, Protzko S, Raghu G, Richeldi L, Sverzellati N, Swigris J, Valeyre D, A. E. C. o. I. I. Pneumonias (2013) An official American Thoracic Society/European Respiratory Society statement: Update of the international multidisciplinary classification of the idiopathic interstitial pneumonias. Am J Respir Crit Care Med 188:733-748. https://doi.org/10.1164/rccm. 201308-1483ST

4. Martinez FJ, Chisholm A, Collard HR, Flaherty KR, Myers J, Raghu G, Walsh SL, White ES, Richeldi L (2017) The diagnosis of idiopathic pulmonary fibrosis: current and future approaches. Lancet Respir Med 5:61-71. https://doi.org/10.1016/ S2213-2600(16)30325-3

5. Walsh SL, Calandriello L, Sverzellati N, Wells AU, Hansell DM, Consort UIPO, U. I. P. O. Consort (2016) Interobserver agreement for the ATS/ERS/JRS/ ALAT criteria for a UIP pattern on CT. Thorax 71:45-51. https://doi.org/10.1136/thoraxjnl2015-207252

6. Erickson BJ, Korfiatis P, Akkus Z, Kline TL (2017) Machine learning for medical imaging. Radiographics 37:505-515. https://doi.org/10.1148/rg. 2017160130

7. Hosny A, Parmar C, Quackenbush J, Schwartz LH, Aerts H (2018) Artificial intelligence in radiology. Nat Rev Cancer 18:500-510. https://doi.org/10. 1038/s41568-018-0016-5

8. Walsh SLF, Calandriello L, Silva M, Sverzellati N (2018) Deep learning for classifying fibrotic lung disease on high-resolution computed tomography: a case-cohort study. Lancet Respir Med 6:837-845. https://doi.org/10.1016/S22132600(18)30286-8

9. Chartrand G, Cheng PM, Vorontsov E, Drozdzal M, Turcotte S, Pal CJ, Kadoury S, Tang A (2017) Deep learning: a primer for radiologists. Radiographics 37:2113-2131. https://doi.org/10.1148/rg. 2017170077 
10. Anthimopoulos M, Christodoulidis S, Ebner L, Christe A, Mougiakakou S (2016) Lung pattern classification for interstitial lung diseases using a deep convolutional neural network. IEEE Trans Med Imaging 35:1207-1216. https://doi.org/10. 1109/TMI.2016.2535865

11. Kim GB, Jung $K H$, Lee $Y$, Kim $H J$, Kim N, Jun $S$, Seo JB, Lynch DA (2018) Comparison of shallow and deep learning methods on classifying the regional pattern of diffuse lung disease. J Digit Imaging 31:415-424. https://doi.org/10.1007/ s10278-017-0028-9

12. Gao M, Bagci U, Lu L, Wu A, Buty M, Shin HC, Roth $\mathrm{H}_{\text {, }}$ Papadakis GZ, Depeursinge A, Summers RM, Xu Z, Mollura DJ (2018) Holistic classification of CT attenuation patterns for interstitial lung diseases via deep convolutional neural networks. Comput Methods Biomech Biomed Eng Imaging Vis 6:1-6. https://doi.org/10.1080/21681163.2015.1124249

13. Raghu G, Collard HR, Egan JJ, Martinez FJ, Behr J, Brown KK, Colby TV, Cordier JF, Flaherty KR, Lasky JA, Lynch DA, Ryu JH, Swigris JJ, Wells AU, Ancochea J, Bouros D, Carvalho C, Costabel U, Ebina M, Hansell DM, Johkoh T, Kim DS, King TE Jr., Kondoh Y, Myers J, Muller NL, Nicholson AG, Richeldi L, Selman M, Dudden RF, Griss BS, Protzko SL, Schunemann HJ, A.E.J.A.C. o.I.P.Fibrosis (2011) An official ATS/ERS/JRS/ALAT statement: idiopathic pulmonary fibrosis: evidence-based guidelines for diagnosis and management. Am J Respir Crit Care Med 183:788-824. https://doi.org/10.1164/rccm. 2009-040GL

14. Christe A, Peters AA, Drakopoulos D, Heverhagen JT, Geiser T, Stathopoulou T, Christodoulidis S, Anthimopoulos M, Mougiakakou SG, Ebner L (2019) Computer-aided diagnosis of pulmonary fibrosis using deep learning and CT images. Invest Radiol 54:627-632. https://doi.org/10.1097/RLI. 0000000000000574

15. Ohkubo H, Nakagawa H, Niimi A (2018) Computerbased quantitative computed tomography image analysis in idiopathic pulmonary fibrosis: A mini review. Respir Investig 56:5-13. https://doi.org/10 1016/j.resinv.2017.10.003

16. Kim HJ, Brown MS, Chong D, Gjertson DW, Lu P, Kim HJ, Coy H, Goldin JG (2015) Comparison of the quantitative $\mathrm{CT}$ imaging biomarkers of idiopathic pulmonary fibrosis at baseline and early change with an interval of 7 months. Acad Radiol 22:70-80. https://doi.org/10.1016/j.acra.2014.08.004

17. Muller NL, Staples CA, Miller RR, Abboud RT (1988) "Density mask". An objective method to quantitate emphysema using computed tomography. Chest 94:782-787. https://doi.org/10.1378/chest.94.4. 782

18. Ohkubo H, Kanemitsu Y, Uemura T, Takakuwa O, Takemura M, Maeno K, Ito Y, Oguri T, Kazawa N, Mikami R, Niimi A (2016) Normal lung quantification in usual interstitial pneumonia pattern: the impact of threshold-based volumetric CT analysis for the staging of idiopathic pulmonary fibrosis. PLoS ONE 11:e152505. https://doi.org/10.1371/ journal.pone.0152505

19. Ash SY, Harmouche R, Vallejo DL, Villalba JA, Ostridge K, Gunville R, Come CE, Onieva Onieva J, Ross JC, Hunninghake GM, El-Chemaly SY, Doyle TJ, Nardelli P, Sanchez-Ferrero GV, Goldberg HJ, Rosas IO, San Jose Estepar R, Washko GR (2017) Densitometric and local histogram based analysis of computed tomography images in patients with idiopathic pulmonary fibrosis. Respir Res 18:45. https://doi.org/10.1186/s12931-017-0527-8

20. Delorme S, Keller-Reichenbecher MA, Zuna I, Schlegel W, Van Kaick G (1997) Usual interstitial pneumonia. Quantitative assessment of highresolution computed tomography findings by computer-assisted texture-based image analysis. Invest Radiol 32:566-574. https://doi.org/10. 1097/00004424-199709000-00009

21. Kim GHJ, Weigt SS, Belperio JA, Brown MS, Shi Y, Lai JH, Goldin JG (2019) Prediction of idiopathic pulmonary fibrosis progression using early quantitative changes on CT imaging for a short term of clinical 18-24-month follow-ups. Eur Radiol. https://doi.org/10.1007/s00330-01906402-6

22. Jacob J, Bartholmai BJ, Rajagopalan S, Kokosi M, Nair A, Karwoski R, Raghunath SM, Walsh SLF, Wells AU, HansellDM (2016) Automated quantitative computed tomography versus visual computed tomography scoring in idiopathic pulmonary fibrosis: validation against pulmonary function. J Thorac Imaging 31:304-311. https://doi.org/10. 1097/rti.0000000000000220

23. Jacob J, Bartholmai BJ, Rajagopalan S, Kokosi M Nair A, Karwoski R, Walsh SL, Wells AU, Hansell DM (2017) Mortality prediction in idiopathic pulmonary fibrosis: evaluation of computer-based $\mathrm{CT}$ analysis with conventional severity measures. Eur Respir J. https://doi.org/10.1183/13993003. 01011-2016

24. Goo JM (2011) A computer-aided diagnosis for evaluating lung nodules on chest CT: the current status and perspective. Korean J Radiol 12:145-155. https://doi.org/10.3348/kjr.2011.12. 2.145

25. Fuehner T, Greer M, Welte T, Gottlieb J (2012) The lung transplant patient in the ICU. Curr Opin Crit Care 18:472-478. https://doi.org/10.1097/MCC. Ob013e328356d982

26. Pehrson LM, Nielsen MB, Ammitzbol Lauridsen C (2019) Automatic pulmonary nodule detection applying deep learning or machine learning algorithms to the LIDC-IDRI database: a systematic review. Diagnostics. https://doi.org/10.3390/ diagnostics 9010029

27. Lodwick GS, Keats TE, Dorst JP (1963) The coding of roentgen images for computer analysis as applied to lung cancer. Radiology 81:185-200. https://doi. org/10.1148/81.2.185

28. Thawani R, McLane M, Beig N, Ghose S, Prasanna P, Velcheti V, Madabhushi A (2018) Radiomics and radiogenomics in lung cancer: a review for the clinician. Cancer Treat Res 115:34-41. https://doi. org/10.1016/j.lungcan.2017.10.015

29. Ather S, Kadir T, Gleeson F (2019) Artificial intelligence and radiomics in pulmonary nodule management: current status and future applications. Clin Radiol. https://doi.org/10.1016/j.crad 2019.04.017

30. Aerts HJ, Grossmann P, Tan Y, Oxnard GR, Rizvi N, Schwartz LH, Zhao B (2016) Defining a radiomic response phenotype: a pilot study using targeted therapy in NSCLC. Sci Rep 6:33860. https://doi.org/ 10.1038/srep33860

31. Rios Velazquez E, Parmar C, Liu Y, Coroller TP, Cruz G, Stringfield O, Ye Z, Makrigiorgos M, Fennessy F, Mak RH, Gillies R, Quackenbush J, Aerts H (2017) Somatic Mutations Drive Distinct Imaging Phenotypes in Lung Cancer. Cancer Res 77:3922-3930. https://doi.org/10.1158/00085472.CAN-17-0122
ONKO-Internetportal

Fokus USA - Neues von den Kongressen ASH und SABCS

Mit Interviews und Gesprächsrunden vom Jahrestreffen der American Society of Hematology (ASH) und dem San Antonio Breast Cancer Symposiums (SABCS) startet das ONKO-Internetportal in das neue Jahr. Mehr als 25.000 Hämatologen aus alle Welt kamen zum ASH-Kongress nach Orlando, um sich über aktuelle Forschungsergebnisse auszutauschen. Bewertungen ausgewählter Daten liefert das ONKO-Internetportal mit einer interdisziplinären Expertenrunde zum Multiplen Myelom und zwei Expertengesprächen zu Lymphomen, Leukämieerkrankungen und MDS. Unter www.krebsgesellschaft.de/ash_2019 analysieren Medizinerinnen und Mediziner die neuen Therapieoptionen und ordnen ihre Relevanz für die klinische Praxis ein. Themen sind u.a. neue vielversprechende Daten zur CAR-T-Zell-Therapie, Studien zur Charakterisierung und Reparatur von Mutationen sowie Kombinationstherapien.

Fast zeitgleich wurden auf dem weltweit größten Brustkrebskongress in San Antonio eine Vielzahl neuer Studienergebnisse vorgestellt. Das ONKO-Internetportal präsentiert Highlights und Kontroversen, u.a. zu den Themen Immuntherapie beim triple-negativen Mammakarzinom, neue Substanzen beim HER2-positiven Brustkrebs und zu den Ergebnissen der PEARLund HER2CLIMB-Studie. Die Expertenrunde Mammakarzinom diskutiert außerdem über die Behandlung mit Aromatase-Hemmern und der

Therapie mit CDK4/6-Inhibitoren. Alle Experten-Videos stehen ab sofort unter www.krebsgesellschaft.de/sabcs_2019 zum Download bereit.

Quelle: ONKO-Internetportal in Kooperation mit der Deutschen Krebsgesellschaft e.V. (DKG), www.krebsgesellschaft.de 